\title{
Constraining exotic spin dependent interactions of muons and electrons
}

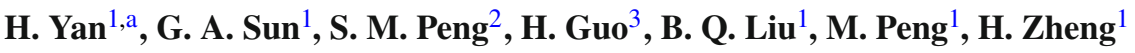 \\ ${ }^{1}$ Key Laboratory of Neutron Physics, Institute of Nuclear Physics and Chemistry, CAEP, Mianyang 621900, Sichuan, China \\ 2 Institute of Nuclear Physics and Chemistry, CAEP, Mianyang 621900, Sichuan, China \\ ${ }^{3}$ Department of Physics, Southeast University, Nanjing 211189, China
}

Received: 9 June 2019 / Accepted: 29 October 2019 / Published online: 25 November 2019

(C) The Author(s) 2019

\begin{abstract}
Many experiments have been performed to search for the exotic spin-dependent interactions in ranges from $\sim \mu \mathrm{m}$ to astrophysical range which corresponds to the energy scale of less than $\sim 10 \mathrm{eV}$. At present, nearly all known experiments searching for these new interactions at the macroscopic range are for protons, neutrons, and electrons. Constraints at this range for other fermions such as muons are scarce, though muons might be the most suspicious particles which might take part in new interactions, considering their involvement of several well-known puzzles of modern physics. We use the anomalous magnetic moment and electric dipole moment (EDM) to study the exotic spin-dependent interactions for muons and electrons. The muon's magnetic moment might indicate existing of the pseudo-scalar-pseudo-scalar (PP) type interaction. We set up a constraint for the scalarpseudo-scalar (SP) type interaction at the interested range for muons. For the PP type interaction of electrons, we obtained a new constraint at the range of $\sim \mathrm{nm}$ to $\sim 1 \mathrm{~mm}$. Since all the present experiments searching for the new forces give zero results, it is reasonable to consider that these new interactions might only couple to muons. We propose to further search for the new interactions using the muon spin rotation techniques.
\end{abstract}

\section{Introduction}

New interactions beyond the Standard Model are possible. Suggested solutions for several important problems of modern physics have led to new interactions mediated by new particles [1]. Exotic macroscopic forces mediated by WISPs

Electronic supplementary material The online version of this article (https://doi.org/10.1140/epjc/s10052-019-7442-8) contains supplementary material, which is available to authorized users.

a e-mail: hyan@caep.cn (weakly-interacting slim particles) is an example. Reference [2] classified the new interactions into 16 different types and most of them are spin dependent. These new interactions have ranges from nanometers to astronomical distance which corresponds to the mediating-boson mass of $\sim 10^{-18} \mathrm{eV}$ to $\sim 100 \mathrm{eV}$. Methods of precision measurement such as the torsion pendulum [3], co-magnetometer of polarized noble gases [4,5], SERF magnetometer [6], polarized ${ }^{3} \mathrm{He}$ atom beam [7], etc are convenient to probe these new interactions at a much lower energy scale than the high energy physics.

According to Refs. [2,8], new interactions between fermions can be induced via the coupling:

$\mathcal{L}_{I}=\bar{\psi}\left(g_{s}+i g_{p} \gamma_{5}\right) \psi \phi$

$\phi$ which mediates the new interaction, is the ALP (AxionLike Particle). To probe the new force is equivalent to detect the ALP which leads to solutions of some very important problems in modern physics. On one hand, axions are possible candidates for the dark matter which remains to be one of the most important unsolved problems in both particle physics and astrophysics [1,9]. On the other hand, axions have attracted a lot of attention in high energy physics since they probably provide the most promising solution to preserve the CP-symmetry in strong interactions [10].

Due to $\mathcal{L}_{I}$, there could be the monopole-monopole, dipole-dipole and monopole-dipole interactions, originated from the SS (Scalar-Scalar), PP (Pseudo-scalar-Pseudoscalar) and SP (Scalar-Pseudo-scalar) coupling respectively and the last two are spin dependent. The SP interaction or the monopole-dipole interaction has attracted much scientific interest recently. At low energy, the interaction between polarized and unpolarized fermions can be expressed as the potential $[2,8]$ :

$V_{S P}(r)=\frac{\hbar^{2} g_{S} g_{P}}{8 \pi m_{1}}\left(\frac{1}{\lambda r}+\frac{1}{r^{2}}\right) \exp (-r / \lambda) \vec{\sigma} \cdot \hat{r}$ 
where $\lambda=\hbar / m_{\phi} c$ is the interaction range, $m_{\phi}$ the mass of the new scalar boson, $\vec{s}=\hbar \vec{\sigma} / 2$ the spin of the polarized fermion, $m_{1}$ the mass of the polarized fermion and $r$ the distance between the interacting particles. The spin-spin dependent potential induced by the PP interaction is:

$$
\begin{aligned}
V_{P P}(r)= & \frac{\hbar^{3} g_{P} g_{P}}{16 \pi c m_{1} m_{2}}\left[\left(\frac{1}{\lambda r^{2}}+\frac{1}{r^{3}}\right) \vec{\sigma}_{1} \cdot \vec{\sigma}_{2}\right. \\
& \left.-\left(\frac{1}{\lambda^{2} r}+\frac{3}{\lambda r^{2}}+\frac{1}{r^{3}}\right)\left(\vec{\sigma}_{1} \cdot \hat{r}\right)\left(\vec{\sigma}_{2} \cdot \hat{r}\right)\right] \\
& \times \exp (-r / \lambda)
\end{aligned}
$$

In order to detect $V_{S P}$, either the source or the probe fermion has to be spin polarized. While to search for $V_{P P}$, both particles need to be polarized. Furthermore, the source and probe fermion have to be close enough which is limited by the force range $\lambda$. Recently, various experiments have been performed or proposed to search for these new interactions which could couple to the spin of the neutron/electron [3-7,11-18]. However, studies of these long-range, spindependent new interactions for other fermions are scarce. Muons are probably the most suspicious fermion which new interactions might be involved. The charge radius puzzles of the muonic hydrogen [19] and deuteron [20] nucleus are wellknown examples [21,22]. Although parity-violating muonic forces mediated by new massive gauge bosons of $\mathrm{MeV}-\mathrm{GeV}$ have been proposed to solve the proton charge radius puzzle, to our best knowledge, no studies have been conducted to search for these new interactions in ranges greater than $\sim \mathrm{nm}$ which corresponds to a mass scale of less than $\sim 100 \mathrm{eV}$ yet.

In this work, we discuss the possibility that the anomalous magnetic moment of the muon might indicate existing of the new SS or PP interaction mediated by ALPs lighter than $\sim 100 \mathrm{eV}$. By using the muon's EDM (Electric Dipole Moment), we could establish a constraint for the parityviolating monopole-dipole interaction mediated by ALPs lighter than $\sim 100 \mathrm{eV}$ for muons. When applying the method to the electrons, constraints of $g_{S}^{e} g_{S}^{e}$ and $g_{P}^{e} g_{P}^{e}$ are obtained for the range between $\sim 1$ and $\sim 1 \mathrm{~mm}$.

\section{The anomalous magnetic moment and EDM induced by new interactions}

The leading order contribution to the electromagnetic vertex from the new interactions is shown in Fig. 1. The new boson line could induce SS, SP and PP type vertexes.

The new interactions, if exist, would not only change the anomalous magnetic moment of the fundamental fermion but also induce the EDM which has been measured for many particles [1]. According to Refs. [23-25], the general formula corresponding to the Feynman diagram shown in Fig. 1 can be expressed as:
Fig. 1 The electromagnetic vertex correction contributed by new interactions

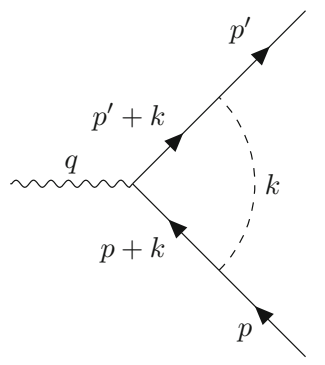

$$
\begin{aligned}
\bar{u}\left(p^{\prime}\right) \Lambda^{\mu} u(p) & \\
= & \bar{u}\left(p^{\prime}\right)\left[\gamma^{\mu} F_{1}\left(q^{2}\right)+i \frac{\sigma^{\mu v} q_{v}}{2 m} F_{2}\left(q^{2}\right)\right. \\
& \left.+\gamma^{5} \frac{\sigma^{\mu \nu} q_{v}}{2 m} F_{3}\left(q^{2}\right)+\gamma^{5}\left(q^{2} \gamma^{\mu}-2 m \gamma^{5} q^{\mu}\right) F_{4}\left(q^{2}\right)\right] u(p)
\end{aligned}
$$

where $q=p^{\prime}-p$. Here $e F_{1}(0)$ gives the renormalized charge, and $F_{4}(0)$ which is called the anapole moment [25], is the possible axial current induced by the new interactions which do not necessarily conserve the parity. These two terms are irrelevant to this work. $\mathrm{e} F_{2}(0) / 2 \mathrm{~m}$ is the anomalous magnetic moment, and $-\mathrm{e} F_{3}(0) / 2 \mathrm{~m}$ the EDM.

Using the well known Gordon decomposition formula and techniques presented in Ref. [26], one can derive the anomalous magnetic moment induced by the SS and PP interaction as [27]

$$
\begin{aligned}
& F_{2}(0)=-\frac{g_{S} g_{S}}{8 \pi^{2}} S S(x) \\
& F_{2}(0)=-\frac{g_{P} g_{P}}{8 \pi^{2}} P P(x)
\end{aligned}
$$

where $S S(x)$ and $P P(x)$ are defined as:

$$
\begin{aligned}
S S(x)= & \frac{3}{2}-x^{2}+x^{2}\left(x^{2}-3\right) \ln x+x\left(x^{2}-1\right) \sqrt{x^{2}-4} \\
& \times\left[\tanh ^{-1}\left(\frac{x}{\sqrt{x^{2}-4}}\right)-\tanh ^{-1}\left(\frac{x^{2}-2}{x \sqrt{x^{2}-4}}\right)\right] \\
P P(x)= & \frac{1}{2}+x^{2}-x^{2}\left(x^{2}-1\right) \ln x+\frac{x^{3}\left(3-x^{2}\right)}{\sqrt{x^{2}-4}} \\
& \times\left[\tanh ^{-1}\left(\frac{x}{\sqrt{x^{2}-4}}\right)-\tanh ^{-1}\left(\frac{x^{2}-2}{x \sqrt{x^{2}-4}}\right)\right] .
\end{aligned}
$$

with $x=m_{\phi} / m$.

EDM can be induced by the possible new interactions. In this case, implementing similar techniques as before, for the SP type new interaction, we obtain [27]

$F_{3}(0)=\frac{g_{S} g_{P}}{4 \pi^{2}} S P(x)$

where

$$
\begin{aligned}
S P(x)= & 1-x^{2} \ln x-\frac{x\left(x^{2}-2\right)}{\sqrt{x^{2}-4}} \\
& \times\left[\tanh ^{-1}\left(\frac{x}{\sqrt{x^{2}-4}}\right)-\tanh ^{-1}\left(\frac{x^{2}-2}{x \sqrt{x^{2}-4}}\right)\right] .
\end{aligned}
$$




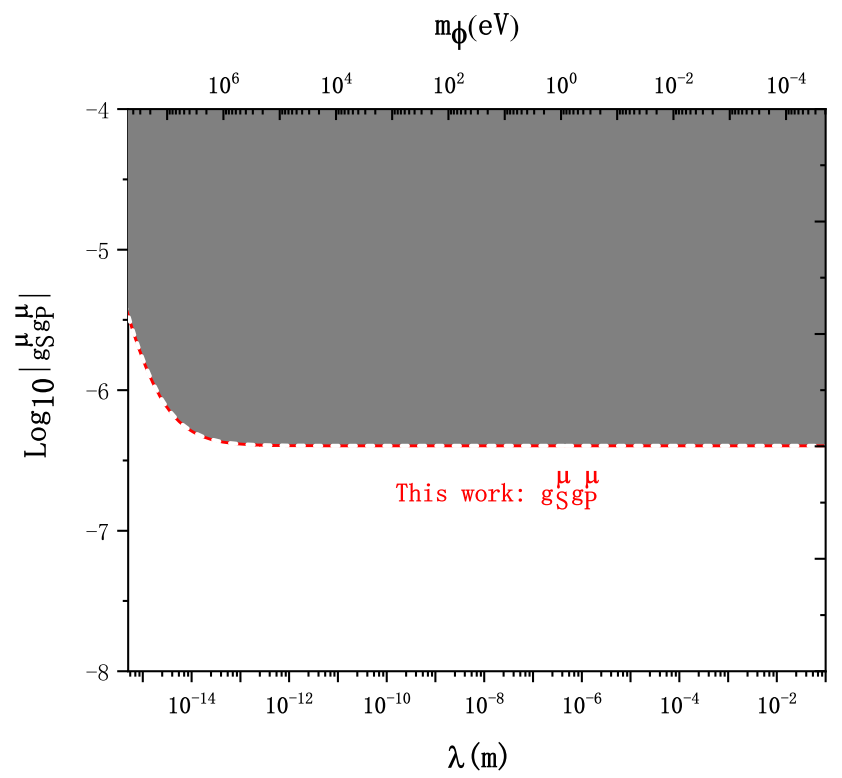

Fig. 2 A constraint to the coupling constant product $\left|g_{S}^{\mu} g_{P}^{\mu}\right|$ as a function of the interaction range $\lambda$ (ALP mass). The (red) dashed line is the result of this work for the muon. The dark grey area is excluded from this work

\section{Applications to the muon and electron}

There is a $\sim 3.7 \sigma[1,28,29]$ difference between the experimental measurement and the theoretical prediction from the Standard Model for the anomalous magnetic moment $a_{\mu}$ of the muon. According to Ref. [30], this difference is explicitly given by

$a_{\mu}^{e x p}-a_{\mu}^{S M}=2.74 \pm 0.73 \times 10^{-9}$

where $a_{\mu}^{\text {exp }}$ is the experimentally measured value and $a_{\mu}^{S M}$ the Standard Model prediction. If the contribution from the long-range new interaction of $V_{P P}$ is considered, then the difference might be explained as a nonzero $g_{S}^{\mu} g_{S}^{\mu}$ or $g_{P}^{\mu} g_{P}^{\mu}$ of:

$$
\begin{aligned}
& \left|g_{S}^{\mu} g_{S}^{\mu}\right|=1.44 \pm 0.38 \times 10^{-7} \\
& \left|g_{P}^{\mu} g_{P}^{\mu}\right|=4.32 \pm 1.16 \times 10^{-7}
\end{aligned}
$$

with $m_{\phi}<100 \mathrm{eV}(\lambda>1 \mathrm{~nm})$.

Plugging in the best known muon EDM [31],

$\left|d_{\mu}\right|<1.8 \times 10^{-19}$ e.cm, 95\% C.L.

a constraint for $g_{S}^{\mu} g_{P}^{\mu}$ can be established as shown in Fig. 2. It is interesting to notice that $g_{P}^{\mu} g_{P}^{\mu}$ is not zero while $g_{S}^{\mu} g_{P}^{\mu}$ is, all at a $\sim 10^{-7}$ level.

We also apply this method to the electron. Theoretically, the anomalous magnetic moment and EDM of the electron can be predicted by the Standard Model [32-34]. Combined with the best known experimental measurement given in Ref.

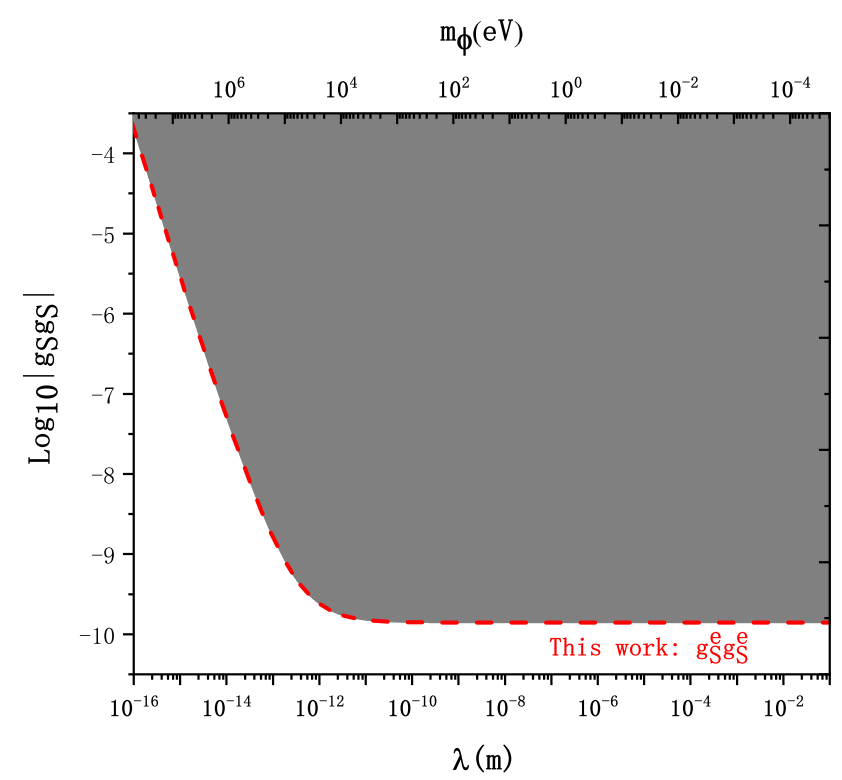

Fig. 3 Constraints to the coupling constant product $\left|g_{S}^{e} g_{S}^{e}\right|$ a function of the interaction range $\lambda$ (ALP mass). The (red) dashed line is the result of this work

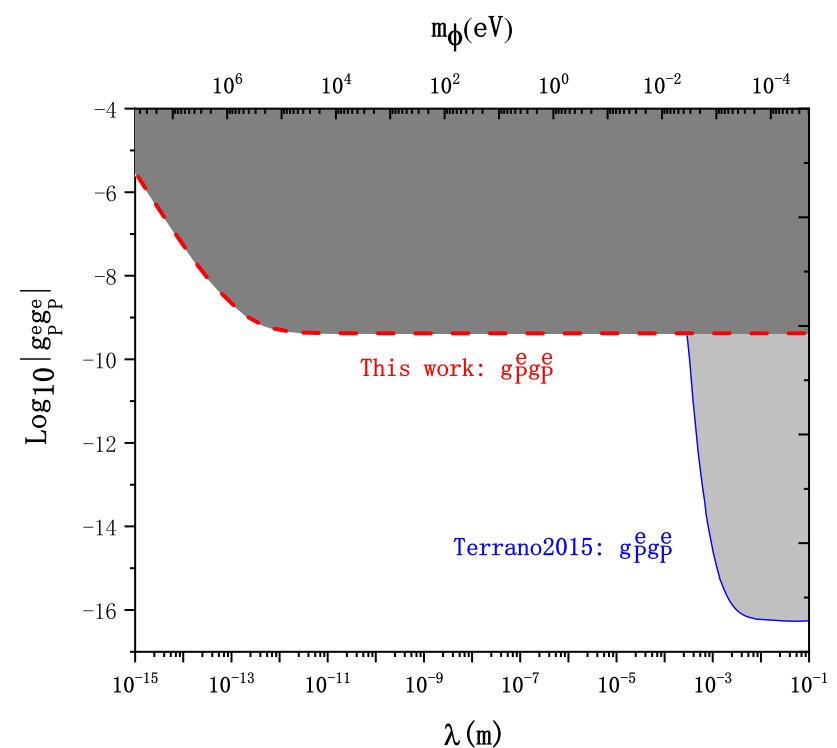

Fig. 4 Constraints to the coupling constant product $\left|g_{S}^{e} g_{P}^{e}\right|$ a function of the interaction range $\lambda$ (ALP mass). The (red) dashed line is the result of this work. The (blue) solid line is the result of Ref. [3]. The dark grey area is excluded by this work and grey area is excluded by Ref. [3]

[35], the possible contributions due to new physics beyond the standard model are

$$
\begin{aligned}
& \left|a_{e}^{e x p}-a_{e}^{S M}\right|<2.66 \times 10^{-12}, 95 \% \text { C.L. } \\
& \left|d_{e}\right|<1.2 \times 10^{-29} \text { e.cm, 95\%C.L. }
\end{aligned}
$$

By using the anomalous magnetic moment, constraints of $\left|g_{S}^{e} g_{S}^{e}\right|$ and $\left|g_{P}^{e} g_{P}^{e}\right|$ for electrons can be established as shown in Figs. 3 and 4, respectively. One can see that this method 
Fig. 5 Schematic of the experimental setup to probe the new spin-dependent interactions for muons. The incoming muon beam which is spin-polarized triggers the clock which defines time zero. A sample is placed close to the muon beam and its polarization can be rotated if new spin-dependent interactions exist. The rotation angle of the muon polarization can be measured from the positron counter

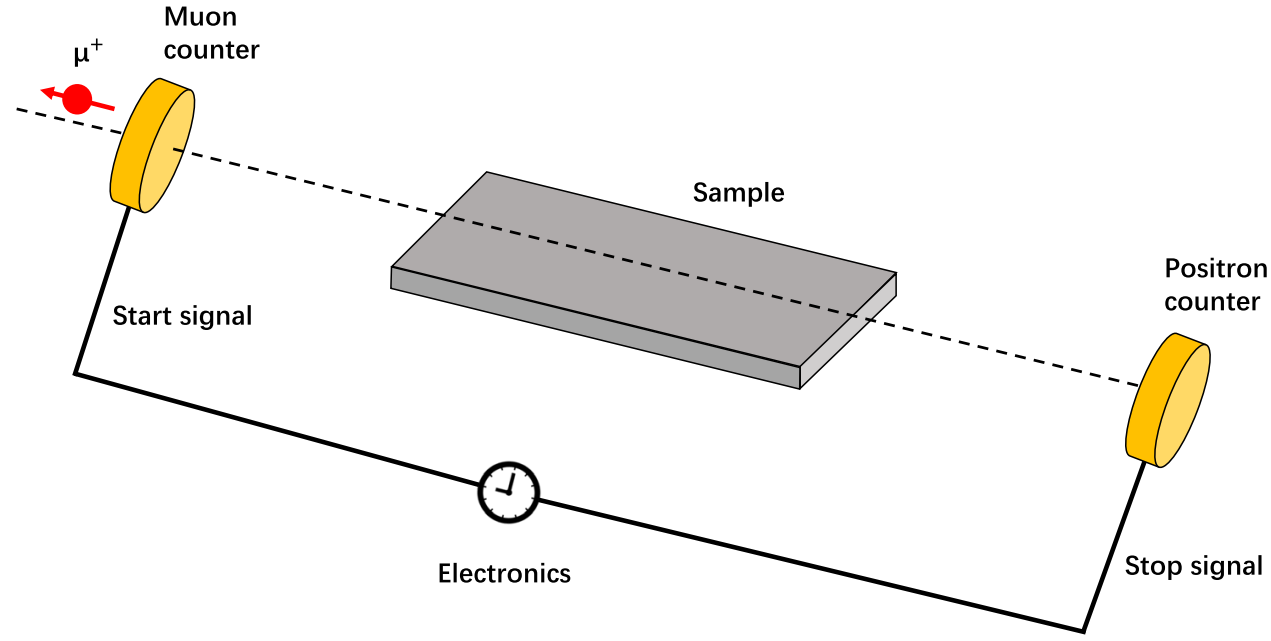

quently used in condensed matter physics [40] and fundamental physics [29]. If a nonmagnetic mass-source can be put in the region close to muon beams as in Fig. 5 [7], constraints on $g_{S} g_{P}^{\mu}$ at long distances can be obtained by measuring changes of the muon polarization. Furthermore, if polarized electron spin-density sources, as the $\mu$-metal shielded $\mathrm{SmCo}_{5}[3,41]$, are used in experiments, then constraints on $g_{P}^{e} g_{P}^{\mu}$ can be established. It is not hard to imagine that the muonic new interactions mediated by light vector particles can also be searched for using experimental schemes as in Ref. [7].

Using the same method, we can also give constraints of $g_{S}^{e} g_{S}^{e}$ and $g_{P}^{e} g_{P}^{e}$ for electrons. This method works at small distances from $\sim \mathrm{nm}$ to $\sim \mathrm{mm}$. Moreover, it can give pure constraints only between the electrons, while many other methods cannot easily isolate the contributions from other fermions like protons or neutrons. Our results for the electron are consistent with zero.

The fact that no new interactions have been detected for electrons, neutrons and protons indicates the muon might be an interesting target for these new forces at long ranges. It is possible that these new interactions might be muonic which means they only couple to the muons. Though studies have performed for muonic new interactions at very short ranges (new boson mass heavier than $\sim \mathrm{MeV}$ ), to the best of our knowledge, no experiments have ever been done to search for these spin-dependent new interactions at long ranges (new boson mass lighter than $\sim 100 \mathrm{eV}$ ).

Acknowledgements We acknowledge support from the National Natural Science Foundation of China, under Grant 91636103, 11675152, 11875238. This work was also supported by National Key Program for Research and Development (Grant 2016YFA0401504). We thank Dr. Queiroz and Dr. Stadnik for providing us useful references. We thank Dr. Stadnik for helpful discussions.

Data Availability Statement This manuscript has no associated data or the data will not be deposited. [Authors' comment: There are no external data associated with the manuscript.] 
Open Access This article is distributed under the terms of the Creative Commons Attribution 4.0 International License (http://creativecomm ons.org/licenses/by/4.0/), which permits unrestricted use, distribution, and reproduction in any medium, provided you give appropriate credit to the original author(s) and the source, provide a link to the Creative Commons license, and indicate if changes were made. Funded by SCOAP ${ }^{3}$.

\section{References}

1. M. Tanabashi et al., Particle Data Group. Phys. Rev. D 98, 030001 (2018)

2. B. Dobrescu, I. Mocioiu, J. High Energy Phys. 11, 005 (2006)

3. W.A. Terrano, E.G. Adelberger, J.G. Lee, B.R. Heckel, Phys. Rev. Lett. 115, 201801 (2015)

4. M. Bulatowicz et al., Phys. Rev. Lett. 111, 102001 (2013)

5. K. Tullney et al., Phys. Rev. Lett. 111, 100801 (2013)

6. G. Vasilakis, J.M. Brown, T.W. Kornack, W. Ketter, M.V. Romalis, Phys. Rev. Lett. 104, 261801 (2009)

7. H. Yan et al., Eur. Phys. J. C 74, 3088 (2014)

8. J.E. Moody, F. Wilczek, Phys. Rev. D 30, 130 (1984)

9. C. Fu et al., Phys. Rev. Lett. 119, 181806 (2017)

10. J.E. Kim, G. Carosi, Rev. Mod. Phys. 82, 557 (2010)

11. S.A. Hoedl, F. Fleischer, E.G. Adelberger, B.R. Heckel, Phys. Rev. Lett. 106, 041801 (2011)

12. G. Raffelt, Phys. Rev. D 86, 015001 (2012)

13. M.P. Ledbetter, M.V. Romalis, D.F.J. Kimball, Phys. Rev. Lett. 110, 040402 (2013)

14. T.M. Leslie, E. Weisman, R. Khatiwada, J.C. Long, Phys. Rev. D 89, 114022 (2014)

15. P.-H. Chu, E. Weisman, C.-Y. Liu, J.C. Long, Phys. Rev. D 91, $102006(2015)$

16. S. Kotler, R. Ozeri, D.F.J. Kimball, Phys. Rev. Lett. 115, 081801 (2015)

17. P.-H. Chu, Y.J. Kim, I. Savukov, Phys. Rev. D 94, 036002 (2016)

18. F. Ficek, D.F.J. Kimball, M.G. Kozlov, N. Leefer, S. Pustelny, D. Budker, Phys. Rev. A 95, 032505 (2017)
19. A. Antognini et al., Science 339, 417 (2013)

20. R. Pohl et al., Science 353, 669 (2016)

21. B. Batell, D. McKeen, M. Pospelov, Phys. Rev. Lett. 107, 011803 (2011)

22. V. Barger, C.W. Chiang, W.Y. Keung, D. Marfatia, Phys. Rev. Lett. 108, 081802 (2012)

23. W. Bernreuther, M. Suzuki, Rev. Mod. Phys. 63, 313 (1991)

24. C. Itzykson, J.-B. Zuber, Quantum Field Theory (McGraw-Hill Inc, New York, 1980)

25. M. Knecht, arXiv:hep-ph/0307239, (2003)

26. A. Zee, Quantum Field Theory in a Nutshell (Princeton University Press, Princeton, 2010)

27. Supplementary Material for "Constraining New Muonic Interactions mediated by Axion-Like-Particles", which includes Refs. [23, 24, 26]

28. M. Lindner, M. Platscher, F.S. Queiroz, arXiv:1610.06587, (2016)

29. A.M. Baldini et al., The MEG Collaboration. Eur. Phys. J. C 76, $223(2016)$

30. T.Blum et al. (RBC and UKQCD Bollaborations), Phys. Rev. Lett. 121, 022003 (2018)

31. G.W. Bennett et al. (Muon (g-2) Collaboration), Phys. Rev. D 80, 052008 (2009)

32. T. Aoyama, M. Hayakawa, T. Kinoshita, M. Nio, Phys. Rev. D 96, 011901(E) (2017)

33. T. Aoyama, M. Hayakawa, T. Kinoshita, M. Nio, Phys. Rev. D 91, $033006(2015)$

34. T. Aoyama, M. Hayakawa, T. Kinoshita, M. Nio, Phys. Rev. Lett. 109, 111807 (2012)

35. The ACME collaboration, Nature 562, 355-360 (2018)

36. X. Rong et al., Nat. Commun. 9, 739 (2018)

37. V.A. Dzuba, V.V. Flambaum, I.B. Samsonov, Y.V. Stadnik, Phys. Rev. D 98, 035048 (2018)

38. Y.V. Stadnik, V.A. Dzuba, V.V. Flambaum, Phys. Rev. Lett. 120, $013202(2018)$

39. Y.V. Stadnik, private communication

40. D. Andreica, Encycl. Condens. Matter Phys. (2005)

41. W. Ji et al., Phys. Rev. Lett. 121, 261803 (2018) 